\title{
Smooth covers of moduli stacks of Riemann surfaces with symmetry
}

\author{
Fabio Perroni ${ }^{1}$
}

Received: 15 December 2020 / Accepted: 20 March 2021 / Published online: 5 April 2021

(c) The Author(s) 2021

\section{Abstract}

We construct explicitly a finite cover of the moduli stack of compact Riemann surfaces with a given group of symmetries by a smooth quasi-projective variety.

\section{Contents}

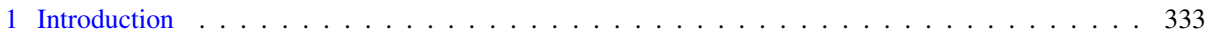

2 Moduli spaces of curves with symmetry . . . . . . . . . . . . . . . . . . . . . . . 334

2.1 On the homology of $M_{g}(G) \ldots \ldots \ldots \ldots \ldots \ldots \ldots \ldots$

3 Level structures and Teichmüller structures . . . . . . . . . . . . . . . . . . . . . . . . . . . 337

3.1 Level structures and Teichmüller structures . . . . . . . . . . . . . . . . . . . . . . . . . 338

4 Smooth covers of $\mathcal{M}_{g}(G, \tau) \ldots \ldots \ldots \ldots \ldots \ldots$

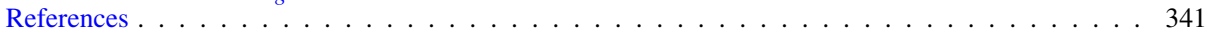

\section{Introduction}

For a finite group $G$, the locus $M_{g}(G)$ (in the moduli space $M_{g}$ ) of curves that have an effective action by $G$ plays an important role in the study of the geometry of $M_{g}$ (for example its singularities [1,2]), in the study of Shimura varieties (see e.g. [3-5] and the references therein), of totally geodesic subvarieties of $M_{g}$ [6], and also in the classification of higher dimensional varieties (see e.g. [7-10]). Similar loci in the moduli space of higher dimensional varieties have been studied in [11].

To investigate the geometry of $M_{g}(G)$ it is more natural to introduce the moduli stack $\mathcal{M}_{g}(G)$ of genus $g$ compact Riemann surfaces with an effective action by $G$. Then we can obtain $M_{g}(G)$ as the image of a finite morphism $\mathcal{M}_{g}(G) \rightarrow M_{g}$. In this paper we study some geometric properties of $\mathcal{M}_{g}(G)$. If $g \geq 2, \mathcal{M}_{g}(G)$ is a complex orbifold, whose connected components are in bijection with the set $\mathbb{T}$ of topological types of the $G$-actions. For any

Dedicated to Fabrizio Catanese on the occasion of his 70th birthday.

$\triangle$ Fabio Perroni

fperroni@units.it

1 Dipartimento di Matematica e Geoscienze, UniversitÀ degli Studi di Trieste, via Valerio 12/1, 34127 Trieste, Italy 
$\tau \in \mathbb{T}$, let $\mathcal{M}_{g}(G, \tau)$ be the corresponding connected component. It is known that $\mathcal{M}_{g}(G, \tau)$ is isomorphic to the stack quotient $\left[\mathcal{T}_{g}^{\tau(G)} / \mathrm{C}_{\Gamma_{g}}(\tau(G))\right]$, where $\mathcal{T}_{g}^{\tau(G)}$ is the locus of points, in the Teichmüller space $\mathcal{T}_{g}$, that are fixed by $\tau(G)$, and $\mathrm{C}_{\Gamma_{g}}(\tau(G))$ is the centralizer of $\tau(G)$ in the mapping class group $\Gamma_{g}$. Using the theory of level structures we define a smooth quasi-projective variety $Z_{\tau}$ such that $\mathcal{M}_{g}(G, \tau) \cong\left[Z_{\tau} / \overline{\mathrm{C}}_{\tau}\right]$, where $\overline{\mathrm{C}}_{\tau}$ is a finite group. Hence $\mathcal{M}_{g}(G, \tau)$ is a smooth Deligne-Mumford stack and $Z_{\tau} \rightarrow \mathcal{M}_{g}(G, \tau)$ is a finite Galois cover. The disjoint union of the $Z_{\tau}$ 's is a finite smooth cover of $\mathcal{M}_{g}(G)$, since $\mathbb{T}$ is finite (see e.g. [12]). Notice that the existence of a smooth quasi-projective variety $Z$ and a finite flat morphism $Z \rightarrow \mathcal{M}_{g}(G)$ follows also from [13].

\section{Moduli spaces of curves with symmetry}

Throughout the article $G$ is a finite group and $g$ is an integer greater or equal than 2 . Let $\mathcal{M}_{g}(G)$ be the stack, in the complex analytic category, whose objects are pairs $(\pi: \mathcal{C} \rightarrow$ $B, \alpha)$, where $\pi: \mathcal{C} \rightarrow B$ is a family of compact Riemann surfaces of genus $g$ and $\alpha: G \times \mathcal{C} \rightarrow$ $\mathcal{C}$ is an effective (holomorphic) action of $G$ on $\mathcal{C}$ such that, for any $a \in G, \pi \circ \alpha\left(a,{ }_{-}\right)=\pi$. A morphism $(\Phi, \varphi):(\pi: \mathcal{C} \rightarrow B, \alpha) \rightarrow\left(\pi^{\prime}: \mathcal{C}^{\prime} \rightarrow B^{\prime}, \alpha^{\prime}\right)$ is a Cartesian diagram

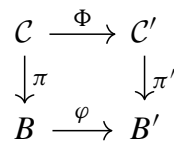

such that, for any $a \in G, \Phi \circ \alpha\left(a, \_\right) \circ \Phi^{-1}=\alpha^{\prime}\left(a, \_\right)$.

Using the Teichmüller space $\mathcal{T}_{g}$, we are going to define a complex orbifold structure on $\mathcal{M}_{g}(G)$. Given a compact, connected, oriented topological surface of genus $g, \Sigma_{g}$, recall that a Teichmüller structure on a Riemann surface $C$ is the isotopy class of an orientation preserving homeomorphism $f: C \rightarrow \Sigma_{g}$, it will be denoted with [ $\left.f\right]$. Two Riemann surfaces with Teichmüller structures $(C,[f]),\left(C^{\prime},\left[f^{\prime}\right]\right)$ are isomorphic, if there exists an isomorphism $F: C \rightarrow C^{\prime}$ such that $[f]=\left[f^{\prime} \circ F\right]$. We will denote with $[C,[f]]$ the class of $(C,[f])$. Then, $\mathcal{T}_{g}$ is the set of isomorphism classes $[C,[f]]$ of compact Riemann surfaces of genus $g$ with Teichmüller structures. The mapping class group of $\Sigma_{g}$, denoted by $\Gamma_{g}$, is the group of all isotopy classes of orientation preserving homeomorphisms of $\Sigma_{g}$. There is a natural action of $\Gamma_{g}$ on $\mathcal{T}_{g}$, given by

$$
[\gamma] \cdot[C,[f]]=[C,[\gamma \circ f]], \quad \forall[\gamma] \in \Gamma_{g},[C,[f]] \in \mathcal{T}_{g} .
$$

Furthermore, for any $[C,[f]] \in \mathcal{T}_{g}$, the homomorphism

$$
\sigma_{[f]}: \operatorname{Aut}(C) \rightarrow \Gamma_{g}, \quad \Phi \mapsto\left[f \circ \Phi \circ f^{-1}\right]
$$

is injective and its image is the stabilizer of $[C,[f]]$ in $\Gamma_{g}$, that we denote by $\operatorname{Stab}_{\Gamma_{g}}([C,[f]])$. We collect in the following theorem several results about the Teichmüller space, for a proof and for more details we refer to [14,15].

Theorem 2.1 $\mathcal{T}_{g}$ has a natural structure of a complex manifold which is homeomorphic to the unit ball in $\mathbb{C}^{3 g-3}$. The action of $\Gamma_{g}$ on $\mathcal{T}_{g}$ is holomorphic and properly discontinuous. The map

$$
\mathcal{T}_{g} \rightarrow M_{g}, \quad[C,[f]] \mapsto[C],
$$


to the coarse moduli space of compact Riemann surfaces of genus $g$ yields an isomorphism $\mathcal{T}_{g} / \Gamma_{g} \cong M_{g}$.

Furthermore there is a universal family of Riemann surfaces of genus $g$ with Teichmüller structure

$$
\eta: \mathcal{X}_{g} \rightarrow \mathcal{T}_{g}
$$

Let $\alpha$ be an effective action of $G$ on $C$, viewed as an injective group homomorphism $\alpha: G \rightarrow \operatorname{Aut}(C)$. Let us choose an orientation preserving homeomorphism $f: C \rightarrow \Sigma_{g}$. Then we have an injective homomorphism

$$
\rho:=\sigma_{[f]} \circ \alpha: G \rightarrow \Gamma_{g}
$$

such that $\rho(G) \subset \operatorname{Stab}_{\Gamma_{g}}([C,[f]])$. Notice that, if $f^{\prime}: C \rightarrow \Sigma_{g}$ is another orientation preserving homeomorphism, we obtain a different homomorphism $\rho^{\prime}: G \rightarrow \Gamma_{g}$. However $[C,[f]]$ and $\left[C,\left[f^{\prime}\right]\right]$ belong to the same $\Gamma_{g}$-orbit, so there exists $[\gamma] \in \Gamma_{g}$ such that $[\gamma] \cdot[C,[f]]=\left[C,\left[f^{\prime}\right]\right]$ and $\rho^{\prime}=[\gamma] \cdot \rho \cdot[\gamma]^{-1}$. This motivates the following definitions (that are already present in the literature, in slightly different forms, see e.g. [12] and the references therein).

Definition 2.2 Let $\alpha: G \rightarrow \operatorname{Aut}(C)$ be an injective homomorphism. Let $\rho$ be defined in (1). The topological type of the $G$-action $\alpha$ is the class of $\rho$ in $\operatorname{Hom}^{\operatorname{inj}}\left(G, \Gamma_{g}\right) / \Gamma_{g}$, where $\operatorname{Hom}^{\mathrm{inj}}\left(G, \Gamma_{g}\right)$ is the set of injective group homomorphisms from $G$ to $\Gamma_{g}$, $\operatorname{Hom}^{\operatorname{inj}}\left(G, \Gamma_{g}\right) / \Gamma_{g}$ is the quotient under the action of $\Gamma_{g}$ by conjugation.

Definition 2.3 The Teichmüller space of compact Riemann surfaces of genus $g$ with $G$ actions is the set

$$
\mathcal{T}_{g}(G)=\left\{([C,[f]], \rho) \in \mathcal{T}_{g} \times \operatorname{Hom}^{\mathrm{inj}}\left(G, \Gamma_{g}\right) \mid \rho(G) \subseteq \operatorname{Stab}_{\Gamma_{g}}([C,[f]])\right\} .
$$

Notice that, if $\operatorname{Hom}^{\operatorname{inj}}\left(G, \Gamma_{g}\right) \neq \emptyset$, then $\mathcal{T}_{g}(G) \neq \emptyset$ by Nielsen realization problem [16] and that it carries the following action by $\Gamma_{g}$ :

$$
[\gamma] \cdot([C,[f]], \rho)=\left([\gamma] \cdot[C,[f]],[\gamma] \cdot \rho \cdot[\gamma]^{-1}\right),
$$

for $[\gamma] \in \Gamma_{g}$ and $([C,[f]], \rho) \in \mathcal{T}_{g}(G)$.

Proposition 2.4 $\mathcal{T}_{g}(G)$ is a complex manifold. Moreover there is an object of $\mathcal{M}_{g}(G)$, $\left(\eta(G): \mathcal{X}_{g}(G) \rightarrow \mathcal{T}_{g}(G), \alpha\right)$, such that the associated classifying morphism $\mathcal{T}_{g}(G) \rightarrow$ $\mathcal{M}_{g}(G)$ induces an isomorphism

$$
\left[\mathcal{T}_{g}(G) / \Gamma_{g}\right] \cong \mathcal{M}_{g}(G),
$$

where $\left[\mathcal{T}_{g}(G) / \Gamma_{g}\right]$ is the stack quotient associated to the action (2). In particular $\mathcal{M}_{g}(G)$ has a structure of complex orbifold in the sense of [17], [15, XII, §4.].

Proof It follows directly from Definition 2.3 that

$$
\mathcal{T}_{g}(G)=\bigsqcup_{\rho \in \operatorname{Hom}^{\operatorname{inj}}\left(G, \Gamma_{g}\right)} \mathcal{T}_{g}^{\rho(G)},
$$

where $\mathcal{T}_{g}^{\rho(G)}$ is the locus of points fixed by $\rho(G)$. Since $\rho(G)$ is a finite group, $\mathcal{T}_{g}{ }^{\rho(G)}$ is a complex submanifold of $\mathcal{T}_{g}$, so the first claim follows.

For any $\rho \in \operatorname{Hom}^{\operatorname{inj}}\left(G, \Gamma_{g}\right)$, let $\eta(\rho): \mathcal{X}_{g}(\rho) \rightarrow \mathcal{T}_{g}^{\rho(G)}$ be the restriction of the universal family. There is a natural effective action, $\alpha(\rho)$, of $G$ on $\mathcal{X}_{g}(\rho)$ such that $\left(\eta(\rho): \mathcal{X}_{g}(\rho) \rightarrow\right.$ 
$\left.\mathcal{T}_{g}^{\rho(G)}, \alpha(\rho)\right)$ is an object of $\mathcal{M}_{g}(G)$. Then we define $\left(\eta(G): \mathcal{X}_{g}(G) \rightarrow \mathcal{T}_{g}(G), \alpha\right)$ as the disjoint union of these objects.

To prove the last statement recall that the objects of $\left[\mathcal{T}_{g}(G) / \Gamma_{g}\right]$, over a base $B$, are pairs $\left(p: P \rightarrow B, f: P \rightarrow \mathcal{T}_{g}(G)\right)$, where $p: P \rightarrow B$ is a principal $\Gamma_{g}$-bundle and $f$ is a $\Gamma_{g}$-equivariant holomorphic map. Let $f^{*}(\eta(G))$ be the pull-back of the family $\eta(G)$. The action of $\Gamma_{g}$ on $P$ extends to a free action on $f^{*}(\eta(G))$. So $f^{*}(\eta(G))$ descends to a family $\pi: \mathcal{C} \rightarrow B$ over $B$. By construction there is a $G$-action, $\alpha$, on $\mathcal{C}$, in such a way that $(\pi: \mathcal{C} \rightarrow B, \alpha)$ is an object of $\mathcal{M}_{g}(G)$. Similarly one associates to every arrow of $\left[\mathcal{T}_{g}(G) / \Gamma_{g}\right]$ an arrow of $\mathcal{M}_{g}(G)$ obtaining an equivalence of categories.

Furthermore we have the following result.

Theorem $2.5 \mathcal{M}_{g}(G)$ is a smooth algebraic Deligne-Mumford stack with quasi-projective coarse moduli space.

A proof of this theorem will be given in Sect. 4, where we define a groupoid presentation of $\mathcal{M}_{g}(G), X_{1} \underset{t}{\stackrel{s}{\rightrightarrows}} X_{0}$, with $X_{0}$ and $X_{1}$ smooth quasi-projective algebraic varieties, and such that $X_{0} \rightarrow \mathcal{M}_{g}(G)$ is finite étale.

\subsection{On the homology of $M_{g}(G)$}

Let $\mathbb{T} \subset \operatorname{Hom}^{\mathrm{inj}}\left(G, \Gamma_{g}\right)$ be a set of representatives of topological types of $G$-actions (see Definition 2.2). Notice that, for any $\tau \in \mathbb{T}$,

$$
\operatorname{Stab}_{\Gamma_{g}}(\tau)=\mathrm{C}_{\Gamma_{g}}(\tau(G)),
$$

where $\mathrm{C}_{\Gamma_{g}}(\tau(G))$ is the centralizer of $\tau(G)$ in $\Gamma_{g}$. Therefore we have an homeomorphism

$$
\mathcal{T}_{g}(G) / \Gamma_{g} \cong \bigsqcup_{\tau \in \mathbb{T}} \mathcal{T}_{g}^{\tau(G)} / \mathrm{C}_{\Gamma_{g}}(\tau(G))
$$

Definition 2.6 Let $\tau \in \mathbb{T}$. The moduli space of compact Riemann surfaces of genus $g$ with $G$ action of topological type $\tau$ is defined as $\mathcal{T}_{g}^{\tau(G)} / \mathrm{C}_{\Gamma_{g}}(\tau(G))$ and will be denoted by $M_{g}(G, \tau)$.

We have the following theorem from [18,19] (see also [20] and the references therein).

Theorem $2.7 \mathcal{T}_{g}^{\tau(G)}$ is bi-holomorphic to $\mathcal{T}_{g^{\prime}, d}$ where $g^{\prime}$ is the genus of $C / G,[C,[f]] \in$ $\mathcal{T}_{g}^{\tau(G)}$, and $d$ is the number of branch points of the quotient map $C \rightarrow C / G$. In particular $\mathcal{T}_{g}^{\tau(G)}$ is homeomorphic to the unit ball in $\mathbb{C}^{3 g^{\prime}-3+d}$.

Let us give an interpretation of the previous theorem. Given a point $[C,[f]] \in \mathcal{T}_{g}^{\tau(G)}$, let $\alpha: \sigma_{[f]}^{-1} \circ \tau: G \rightarrow \operatorname{Aut}(C)$. Then $f:(C, \alpha) \rightarrow\left(\Sigma_{g}, f \circ \alpha \circ f^{-1}\right)$ is $G$-equivariant, so it yields an orientation-preserving homeomorphism of $\theta: C^{\prime} / G \rightarrow \Sigma_{g}^{\prime} / G$, where $C^{\prime} \subseteq C$ and $\Sigma_{g}^{\prime} \subseteq \Sigma_{g}$ are the loci of points with trivial stabilizer. The bi-holomorphism in Theorem 2.7 sends $[C,[f]]$ to $\left[\Sigma_{g}^{\prime} / G,[\theta]\right] \in \mathcal{T}_{g^{\prime}, d}$.

We describe now the map $\mathcal{T}_{g^{\prime}, d} \rightarrow M_{g}(G, \tau)$, which is the composition of the inverse of the previous one, $\mathcal{T}_{g^{\prime}, d} \rightarrow \mathcal{T}_{g}^{\tau(G)}$, with the quotient $\mathcal{T}_{g}^{\tau(G)} \rightarrow \mathcal{T}_{g}^{\tau(G)} / \mathrm{C}_{\Gamma_{g}}(\tau(G))$. Let $p: \Sigma_{g} \rightarrow$ $\Sigma_{g} / G$ be the quotient map, let $y \in \Sigma_{g}^{\prime} / G$, and let $x \in p^{-1}(y)$. Let $\mu: \pi_{1}\left(\Sigma_{g}^{\prime} / G, y\right) \rightarrow$ 
$G$ be the monodromy of the covering $p$ restricted to $\Sigma_{g}^{\prime}$. For any $\left[D^{\prime},[\theta]\right] \in \mathcal{T}_{g^{\prime}, d}$ the composition

$$
\mu \circ \theta_{*}: \pi_{1}\left(D^{\prime}, \theta^{-1}(y)\right) \rightarrow G
$$

gives a $G$-cover $C^{\prime} \rightarrow D^{\prime}$, hence a point $[C, \alpha] \in M_{g}(G, \tau)$. Then the map $\mathcal{T}_{g^{\prime}, d} \rightarrow$ $M_{g}(G, \tau)$ sends $\left[D^{\prime},[\theta]\right]$ to $[C, \alpha]$.

Notice that the class of $\mu$,

$$
[\mu] \in \operatorname{Hom}\left(\pi_{1}\left(\Sigma_{g}^{\prime} / G, y\right), G\right) / G,
$$

where $G$ acts by conjugation, does not depend from the choice of $y$, and that $\Gamma_{g^{\prime}, d}$ acts on $\operatorname{Hom}\left(\pi_{1}\left(\Sigma_{g}^{\prime} / G, y\right), G\right) / G$.

It follows from this that

$$
M_{g}(G, \tau) \cong \mathcal{T}_{g^{\prime}, d} / \operatorname{Stab}_{\Gamma_{g^{\prime}, d}}([\mu]) .
$$

In particular the homology of $M_{g}(G, \tau)$ with rational coefficients can be computed as the homology of the group $\operatorname{Stab}_{\Gamma_{g^{\prime}, d}}([\mu])$ :

$$
H_{n}\left(M_{g}(G, \tau) ; \mathbb{Q}\right) \cong H_{n}\left(\operatorname{Stab}_{\Gamma_{g^{\prime}, d}}([\mu]) ; \mathbb{Q}\right) .
$$

Let now $\left(\alpha_{1}, \beta_{1}, \ldots, \alpha_{g^{\prime}}, \beta_{g^{\prime}}, \gamma_{1}, \ldots, \gamma_{d}\right)$ be a geometric basis of the fundamental group $\pi_{1}\left(\Sigma_{g^{\prime}} \backslash\left\{y_{1}, \ldots, y_{d}\right\}, y\right)$ (here we follow the notation of [12]). Setting $a_{i}=\mu\left(\alpha_{i}\right), b_{i}=$ $\mu\left(\beta_{i}\right), c_{j}=\mu\left(\gamma_{j}\right)$, we obtain an element

$$
\left[\left(a_{1}, b_{1}, \ldots, a_{g^{\prime}}, b_{g^{\prime}}, c_{1}, \ldots, c_{d}\right)\right] \in G^{2 g^{\prime}+d} / G,
$$

where $G$ acts diagonally by conjugation. This yields an injective correspondence between classes $[\mu]$ of monodromies and elements of $G^{2 g^{\prime}+d} / G$. $\Gamma_{g^{\prime}, d}$ acts on $G^{2 g^{\prime}+d} / G$ (see e.g. $\left[12\right.$, Sec 2.]) and the homology of $\operatorname{Stab}_{\Gamma_{g^{\prime}, d}}([\mu])$ is isomorphic to the equivariant homology of the orbit of $\left[\left(a_{1}, b_{1}, \ldots, a_{g^{\prime}}, b_{g^{\prime}}, c_{1}, \ldots, c_{d}\right)\right]$ under the action of $\Gamma_{g^{\prime}, d}$. This motivates the following

Question 2.8 [21] Let $n, d \in \mathbb{N}$. Are there constants $a, b$ such that the dimension of $H_{n}^{\Gamma_{g^{\prime}, d}}\left(G^{2 g^{\prime}+d} / G ; \mathbb{Q}\right)$ is independent of $g^{\prime}$, in the range $g^{\prime}>a n+b$ ?

We proved in [12] that the previous question has an affermative answer in the case where $n=0$.

\section{Level structures and Teichmüller structures}

In this section we recall some basic results and we fix the notation, for the proofs and for more details we refer to [15, Chapter XVI]. Given two groups $G_{1}$ and $G_{2}$ we denote with $\operatorname{Hom}\left(G_{1}, G_{2}\right)$ the set of homomorphisms from $G_{1}$ to $G_{2}$. Notice that there is an action of $G_{2}$ on $\operatorname{Hom}\left(G_{1}, G_{2}\right)$, which is induced by the action of $G_{2}$ on itself by conjugation $\left((g, h) \mapsto g^{-1} h g\right)$. An exterior group homomorphism from $G_{1}$ to $G_{2}$ is an element of the quotient set $\operatorname{Hom}\left(G_{1}, G_{2}\right) / G_{2}$. For any $\varphi \in \operatorname{Hom}\left(G_{1}, G_{2}\right)$ we denote with $\hat{\varphi}$ its class in $\operatorname{Hom}\left(G_{1}, G_{2}\right) / G_{2}$. We will say that $\hat{\varphi}$ is an exterior group monomorphism (respectively epimorphism, isomorphism) if $\varphi$ is a monomorphism (respectively epimorphism, isomorphism). 
Let $X$ be a path connected topological space. For any pair of points $x, y \in X$ and for any continuous path $\gamma$ from $x$ to $y$, let $\varphi_{\gamma}: \pi_{1}(X, x) \rightarrow \pi_{1}(X, y)$ be the isomorphism that sends any $[c] \in \pi_{1}(X, x)$ to $\left[\gamma^{-1} \cdot c \cdot \gamma\right]$, where $c$ is a loop in $X$ based at $x$ and $[c]$ is its homotopy class.

Let $H$ be a group. We will identify two exterior homomorphisms $\hat{\alpha} \in \operatorname{Hom}\left(\pi_{1}(X, x), H\right) / H$ and $\hat{\beta} \in \operatorname{Hom}\left(\pi_{1}(X, y), H\right) / H$ if $\hat{\alpha}=\widehat{\beta \circ \varphi_{\gamma}}$. Notice that this definition does not depend on the choice of $\gamma$ and yields an equivalence relation on the disjoint union

$$
\bigsqcup_{x \in X} \operatorname{Hom}\left(\pi_{1}(X, x), H\right) / H .
$$

The class of $\hat{\alpha}$ will be denoted with $[\alpha]$.

Definition 3.1 A Teichmüller structure of level $H$ on $X$ is the class $[\alpha]$ of an exterior epimorphism $\hat{\alpha} \in \operatorname{Hom}\left(\pi_{1}(X, x), H\right) / H$, for $x \in X$.

Let now $C$ and $C^{\prime}$ be two compact Riemann surfaces. Let $[\alpha]$ and $\left[\alpha^{\prime}\right]$ be two Teichmüller structures of level $H$ on $C$ and $C^{\prime}$, respectively. We say that the pairs $(C,[\alpha])$ and $\left(C^{\prime},\left[\alpha^{\prime}\right]\right)$ are isomorphic if there exists an isomorphism $F: C \rightarrow C^{\prime}$ such that $[\alpha]=\left[\alpha^{\prime} \circ F_{*}\right]$, where $F_{*}: \pi_{1}(C, x) \rightarrow \pi_{1}\left(C^{\prime}, F(x)\right)$ is the homomorphism induced by $F, \alpha: \pi_{1}(C, x) \rightarrow H$ and $\alpha^{\prime}: \pi_{1}\left(C^{\prime}, F(x)\right) \rightarrow H$ are representatives of $[\alpha]$ and $\left[\alpha^{\prime}\right]$, respectively. The isomorphism class of $(C,[\alpha])$ will be denoted $[C ; \alpha]$. The set of isomorphism classes of compact Riemann surfaces of genus $g$ with Teichmüller structures of level $H$ will be denoted ${ }_{H} M_{g}$. When $g \geq 2$ it is possible to define a structure of complex analytic space on ${ }_{H} M_{g}$, called the moduli space of genus $g$ curves with Teichmüller structure of level $H$. We refer to [15] for more details.

\subsection{Level structures and Teichmüller structures}

Let $[\psi]$ be a Teichmüller structure of level $H$ on $\Sigma_{g}$. The following map defines a morphism of complex analytic spaces:

$$
t_{[\psi]}: \mathcal{T}_{g} \rightarrow{ }_{H} M_{g}, \quad[C,[f]] \mapsto\left[C ; \psi \circ f_{*}\right],
$$

where $f$ is a representative of $[f]$ and $\psi: \pi_{1}\left(\Sigma_{g}, f(x)\right) \rightarrow H$ is a representative of $[\psi]$.

Remark 3.2 As explained in [15], the mapping class group $\Gamma_{g}$ acts on the set of Teichmüller structures of level $H$ on $\Sigma_{g}$ as follows: $[\psi] \cdot[\gamma]=\left[\psi \circ \gamma_{*}\right]$. Moreover every connected component of ${ }_{H} M_{g}$ coincides with $t_{[\psi]}\left(\mathcal{T}_{g}\right)$, for some $[\psi]$, we denote $t_{[\psi]}\left(\mathcal{T}_{g}\right)$ by $M_{g}[\psi]$. Hence we have the following decomposition,

$$
{ }_{H} M_{g}=\coprod_{[\psi] \bmod \Gamma_{g}} M_{g}[\psi] .
$$

Let $\Lambda_{[\psi]}:=\left\{[\gamma] \in \Gamma_{g} \mid[\psi] \cdot[\gamma]=[\psi]\right\}$. Then

$$
M_{g}[\psi]=\mathcal{T}_{g} / \Lambda_{[\psi]} \cdot
$$

Furthermore, if $\Lambda_{[\psi]}$ is a normal subgroup of $\Gamma_{g}$ and $\Gamma_{g}[\psi]:=\Gamma_{g} / \Lambda_{[\psi]}$, then

$$
M_{g}=M_{g}[\psi] / \Gamma_{g}[\psi] .
$$

We report the following result from [15], where a characteristic subgroup of $\pi_{1}\left(\Sigma_{g}, x\right)$ is a subgroup that is mapped to itself by every automorphism of $\pi_{1}\left(\Sigma_{g}, x\right)$.

Lemma 3.3 If $\operatorname{ker}(\psi)$ is a characteristic subgroup, then $\Lambda_{[\psi]}$ is a normal subgroup of $\Gamma_{g}$. 
Proof Under our hypotheses any automorphism $\gamma$ of $\pi_{1}\left(\Sigma_{g}\right)$ induces an automorphism $\bar{\gamma}$ of $H$ such that $\psi \circ \gamma=\bar{\gamma} \circ \psi$. This yields a group homomorphism $\operatorname{Out}^{+}\left(\pi_{1}\left(\Sigma_{g}\right)\right) \rightarrow \operatorname{Out}(H)$, $[\gamma] \mapsto[\bar{\gamma}]$, whose kernel is $\Lambda_{[\psi]}$, under the identification of $\operatorname{Out}^{+}\left(\pi_{1}\left(\Sigma_{g}\right)\right)$ with $\Gamma_{g}$.

In the case where $\operatorname{ker}(\psi)$ is a characteristic subgroup we can describe the action of $\Gamma_{g}$ on $M_{g}[\psi]$ as follows [15].

Lemma 3.4 Let $\operatorname{ker}(\psi)$ be a characteristic subgroup of $\pi_{1}\left(\Sigma_{g}\right)$. Then $\Gamma_{g}$ acts on $M_{g}[\psi]$ as follows:

$$
[\gamma] \cdot[C ; \alpha]=[C ; \bar{\gamma} \circ \alpha],
$$

where $\bar{\gamma}$ is the automorphism of $H$ defined in the proof of Lemma 3.3.

Let now $m \in \mathbb{Z}_{\geq 1}$ and let $\chi_{m}: \pi_{1}\left(\Sigma_{g}\right) \rightarrow H_{1}\left(\Sigma_{g}, \mathbb{Z} / m \mathbb{Z}\right)$ be the composition of the natural morphism $\pi_{1}\left(\Sigma_{g}\right) \rightarrow H_{1}\left(\Sigma_{g}, \mathbb{Z}\right)$ with the reduction modulo $m$. Then $H_{1}\left(\Sigma_{g}, \mathbb{Z} / m \mathbb{Z}\right)$ is a strongly characteristic quotient of $\pi_{1}\left(\Sigma_{g}\right)$ (i.e. there is only one subgroup $K$ of $\pi_{1}\left(\Sigma_{g}\right)$ such that $\pi_{1}\left(\Sigma_{g}\right) / K$ is isomorphic to $H_{1}\left(\Sigma_{g}, \mathbb{Z} / m \mathbb{Z}\right)$ ), in particular $\operatorname{ker}\left(\chi_{m}\right)$ is a characteristic subgroup of $\pi_{1}\left(\Sigma_{g}\right)$. In this situation we use the following notation:

$$
M_{g}[m]:=M_{g}\left[\chi_{m}\right], \quad \Gamma_{g}[m]:=\Gamma_{g}\left[\chi_{m}\right], \quad \Lambda_{[m]}:=\Lambda_{\left[\chi_{m}\right]}, \quad t_{[m]}:=t_{\left[\chi_{m}\right]} .
$$

Furthermore we have that

$$
\Gamma_{g}[m] \cong \mathrm{Sp}_{2 g}(\mathbb{Z} / m \mathbb{Z})
$$

and moreover $M_{g}[m]$ coincides with the set of isomorphism classes of pairs $(C, \rho)$, where $\rho: H_{1}(C, \mathbb{Z} / m \mathbb{Z}) \rightarrow(\mathbb{Z} / m \mathbb{Z})^{2 g}$ is a symplectic isomorphism, with respect to the intersection form on $H_{1}(C, \mathbb{Z} / m \mathbb{Z})$ and the standard symplectic form on $(\mathbb{Z} / m \mathbb{Z})^{2 g}$.

\section{Smooth covers of $\mathcal{M}_{g}(G, \tau)$}

Let $\tau: G \rightarrow \Gamma_{g}$ be an injective homomorphism, we denote with $\mathrm{C}_{\tau}$ the centralizer of $\tau(G)$ in $\Gamma_{g}$, and with $\mathrm{N}_{\tau}$ the normalizer of $\tau(G)$ in $\Gamma_{g}$. For any subgroup $H \leq \Gamma_{g}$, its image under the quotient morphism $\Gamma_{g} \rightarrow \Gamma_{g}[m]=\Gamma_{g} / \Lambda_{[m]}$ will be denoted by $\bar{H}$.

Proposition 4.1 Let $m \geq 3$ be an integer and $Z_{\tau}:=\mathcal{T}_{g}^{\tau(G)} /\left(\Lambda_{[m]} \cap \mathrm{C}_{\tau}\right)$. Then $Z_{\tau}$ is a complex manifold and, for $M_{g}(G, \tau)$ defined in Definition 2.6, the quotient morphism $\mathcal{T}_{g}^{\tau(G)} \rightarrow M_{g}(G, \tau)$ induces a finite morphism

$$
Z_{\tau} \rightarrow M_{g}(G, \tau),
$$

which gives an isomorphism between $M_{g}(G, \tau)$ and $Z_{\tau} / \overline{\mathrm{C}}_{\tau}$.

Proof The first claim follows from the fact that $\Lambda_{[m]}$ acts freely on $\mathcal{T}_{g}$, since $m \geq 3$. To see this, let $[\gamma] \in \Lambda_{[m]}$ and let $[C,[f]] \in \mathcal{T}_{g}$ such that $[\gamma] \cdot[C,[f]]=[C,[f]]$. Then $[\gamma] \in \operatorname{Aut}(C)$. Let $\bar{\gamma}_{*}$ be the induced automorphism of $H_{1}(C, \mathbb{Z} / m \mathbb{Z})$. Since $[\gamma] \in \Lambda_{[m]}$, $\bar{\gamma}_{*}=\mathrm{Id}$, then $[\gamma]=\operatorname{Id}_{C}[15$, Prop. (2.8), p. 512].

The last claim follows from the fact that $\Lambda_{[m]}$ is a normal subgroup of $\Gamma_{g}$ (Lemma 3.3) and $\overline{\mathrm{C}}_{\tau}=\frac{\mathrm{C}_{\tau}}{\mathrm{C}_{\tau} \cap \Lambda_{[m]}}$, so

$$
M_{g}(G, \tau)=\mathcal{T}_{g}^{\tau(G)} / \mathrm{C}_{\tau}=Z_{\tau} / \overline{\mathrm{C}}_{\tau}
$$


Now we prove that $Z_{\tau}$ is a quasi-projective algebraic variety. Although this fact can be deduced from [20] or [2], we give here, for completeness, an elementary proof by showing that there is a finite morphism $Z_{\tau} \rightarrow\left(M_{g}[m]\right)^{\overline{\tau(G)}}$, where $\left(M_{g}[m]\right)^{\overline{\tau(G)}}$ is the set of points of $M_{g}[m]$ fixed by the action of $\overline{\tau(G)}$. By a finite morphism of complex analytic spaces we mean a proper morphism with finite fibers.

Proposition 4.2 Let $t_{[m]}: \mathcal{T}_{g} \rightarrow M_{g}[m]$ be the morphism defined in Sect. 3.1, $m \geq 3$. Then the following statements hold true:

1. $t_{[m]}\left(\mathcal{T}_{g}^{\tau(G)}\right)=\left(M_{g}[m]\right)^{\overline{\tau(G)}}$;

2. let $t_{[m] \mid}$ be the restriction of $t_{[m]}$ to $\mathcal{T}_{g}^{\tau(G)}$, then the following diagram is commutative:

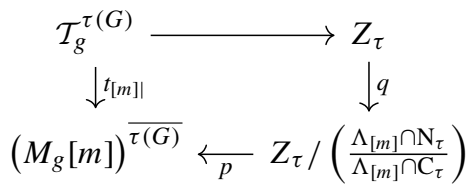

where the horizontal arrow to the top is the quotient map by $\Lambda_{[m]} \cap \mathrm{C}_{\tau}, q$ is the quotient map by $\frac{\Lambda_{[m]} \cap N_{\tau}}{\Lambda_{[m]} \cap C_{\tau}}$ and p sends any equivalence class of the $\left(\Lambda_{[m]} \cap N_{\tau}\right)$-action to the corresponding equivalence class of the $\Lambda_{[m]}$-action (under the identification of $Z_{\tau} /\left(\frac{\Lambda_{[m]} \cap N_{\tau}}{\Lambda_{[m]} \cap C_{\tau}}\right)$ with $\left.\frac{\mathcal{T}_{g}^{\tau(G)}}{\Lambda_{[m]} \cap \mathrm{N}_{\tau}}\right)$;

3. the morphism $p \circ q$ is finite, therefore $Z_{\tau}$ has a structure of complex quasi-projective algebraic variety and $p \circ q$ is algebraic.

Proof 1. Since $\Lambda_{[m]}$ is normal in $\Gamma_{g}$ (Lemma 3.3), $t_{[m]}$ is $G$-equivariant, therefore $t_{[m]}\left(\mathcal{T}_{g}^{\tau(G)}\right) \subseteq\left(M_{g}[m]\right)^{\overline{\tau(G)}}$. On the other hand, for any given $\left[C, \chi_{m} \circ f_{*}\right] \in$ $\left(M_{g}[m]\right)^{\overline{\tau(G)}}$ and $[\gamma] \in \tau(G)$, from the equality

$$
\left[C, \chi_{m} \circ f_{*}\right]=\overline{[\gamma]} \cdot\left[C, \chi_{m} \circ f_{*}\right]
$$

it follows that $[C,[f]]$ and $[\gamma] \cdot[C,[f]]$ map to the same point of $M_{g}[m]$. So, if $[\gamma] \notin$ $\operatorname{Stab}_{\Gamma_{g}}([C,[f]])$, it would be an element of finite order of $\Lambda_{[m]}$, but this contradicts the fact that $\Lambda_{[m]}$ acts freely on $\mathcal{T}_{g}$.

The statement in 2) follows from the fact that $M_{g}[m]=t_{[m]}\left(\mathcal{T}_{g}\right)=\mathcal{T}_{g} / \Lambda_{[m]}$ and from 1). To prove 3), we show that $p \circ q$ is closed and has finite fibers. Let us first show that $q$ has finite fibers, equivalently that $\Lambda_{[m]} \cap \mathrm{C}_{\tau}$ has finite index in $\Lambda_{[m]} \cap \mathrm{N}_{\tau}$. Notice that, for $\tau(G)=\left\{h_{1}, \ldots, h_{|\tau(G)|}\right\}, \mathrm{C}_{\tau}$ is the stabilizer of $\left(h_{1}, \ldots, h_{|\tau(G)|}\right) \in \prod_{h \in \tau(G)} \tau(G)$ with respect to the action of $\mathrm{N}_{\tau}$ given by conjugation on each factor. So $\left[\mathrm{N}_{\tau}: \mathrm{C}_{\tau}\right]<\infty$, hence also $\left[\Lambda_{[m]} \cap \mathrm{N}_{\tau}: \Lambda_{[m]} \cap \mathrm{C}_{\tau}\right]<\infty$. To see that $p$ has finite fibers, notice that for any $\left[C,\left[\chi_{m} \circ f_{*}\right]\right] \in\left(M_{g}[m]\right)^{\overline{\tau(G)}}$

$$
p^{-1}\left(\left[C,\left[\chi_{m} \circ f_{*}\right]\right]\right)=\frac{\left(\Lambda_{[m]} \cdot[C,[f]]\right) \cap \mathcal{T}_{g}^{\tau(G)}}{\Lambda_{[m]} \cap \mathrm{N}_{\tau}},
$$

and, since $\Lambda_{[m]}$ acts freely on $\mathcal{T}_{g}^{\tau(G)}$ (see the proof of Proposition 4.1), $p^{-1}\left(\left[C,\left[\chi_{m} \circ f_{*}\right]\right]\right)$ is in bijection with

$$
\frac{\left\{\lambda \in \Lambda_{[m]} \mid \lambda^{-1} \tau(G) \lambda \subseteq \operatorname{Stab}_{\Gamma_{g}}([C,[f]])\right\}}{\Lambda_{[m]} \cap \mathrm{N}_{\tau}} .
$$


The claim follows since the map from the quotient set in (4) to the set of subgroups of $\operatorname{Stab}_{\Gamma_{g}}([C,[f]])$, induced by $\lambda \mapsto \lambda^{-1} \tau(G) \lambda$, is injective.

The fact that $p \circ q$ is closed follows from the fact that, for any closed subset $A \subseteq \mathcal{T}_{g}^{\tau(G)}$, the union of all $[\gamma] \cdot A,[\gamma] \in \Gamma_{g}$, is closed in $\mathcal{T}_{g}$ (see e.g. [20, proof of Theorem 1]). Finally, $Z_{\tau}$ and $p \circ q$ are algebraic by the Generalized Riemann Existence Theorem of Grauert-Remmert [22] (see [[23], Theorem 3.2, Appendix B]), $Z_{\tau}$ is quasi-projective since $\left(M_{g}[m]\right)^{\overline{\tau(G)}}$ is so.

Proof of Thm. 2.5 From the proof of Proposition 2.4 we have that

$$
\mathcal{M}_{g}(G)=\bigsqcup_{\tau \in \mathbb{T}}\left[\mathcal{T}_{g}^{\tau(G)} / \mathrm{C}_{\tau}\right],
$$

where we use the notation of Sect. 2.1. Since $\Lambda_{[m]} \cap \mathrm{C}_{\tau}$ acts freely on $\mathcal{T}_{g}^{\tau(G)}$ the stack quotient $\left[\mathcal{T}_{g}^{\tau(G)} /\left(\Lambda_{[m]} \cap \mathrm{C}_{\tau}\right)\right]$ is represented by the variety $Z_{\tau}=\mathcal{T}_{g}{ }^{\tau(G)} /\left(\Lambda_{[m]} \cap \mathrm{C}_{\tau}\right)$, hence

$$
\mathcal{M}_{g}(G)=\bigsqcup_{\tau \in \mathbb{T}}\left[Z_{\tau} / \overline{\mathrm{C}}_{\tau}\right]
$$

The claim follows from the fact that the stack quotient of the algebraic variety $Z_{\tau}$ (Proposition 4.1) by the finite group $\overline{\mathrm{C}}_{\tau}$ is a Deligne-Mumford stack (see e.g. [24, (4.6.1)]).

Acknowledgements I would like to thank Fabrizio Catanese for introducing me to the subject of moduli spaces of curves with symmetries, and for teaching me a lot about this. I am grateful to Paola Frediani and Alessandro Ghigi for discussions that motivated the present article. The research was partially supported by the national projects PRIN 2015EYPTSB-PE1 "Geometria delle varietà algebriche" and 2017SSNZAW 005-PE1 "Moduli Theory and Birational Classification", by the research group GNSAGA of INDAM and by FRA of the University of Trieste.

Funding Open access funding provided by Università degli Studi di Trieste within the CRUI-CARE Agreement.

Open Access This article is licensed under a Creative Commons Attribution 4.0 International License, which permits use, sharing, adaptation, distribution and reproduction in any medium or format, as long as you give appropriate credit to the original author(s) and the source, provide a link to the Creative Commons licence, and indicate if changes were made. The images or other third party material in this article are included in the article's Creative Commons licence, unless indicated otherwise in a credit line to the material. If material is not included in the article's Creative Commons licence and your intended use is not permitted by statutory regulation or exceeds the permitted use, you will need to obtain permission directly from the copyright holder. To view a copy of this licence, visit http://creativecommons.org/licenses/by/4.0/.

\section{References}

1. Cornalba, M.: On the locus of curves with automorphisms. Ann. Mat. Pura Appl. 149(4), 135-151 (1987)

2. Catanese, F.: Irreducibility of the Space of Cyclic Covers of Algebraic Curves of Fixed Numerical Type and the Irreducible Components of Sing $\left(\overline{\mathfrak{M}}_{g}\right)$, Advances in Geometric Analysis, Adv. Lect. Math. (ALM), vol. 21, pp. 281-306. Int. Press, Somerville (2012)

3. Frediani, P., Penegini, M., Porru, P.: Shimura varieties in the Torelli locus via Galois coverings of elliptic curves. Geom. Dedicata 181, 177-192 (2016)

4. Mohajer, A., Zuo, K.: On Shimura subvarieties generated by families of abelian covers of $\mathbb{P}^{1}$. J. Pure Appl. Algebra 222(4), 931-949 (2018)

5. Colombo, E., Frediani, P., Ghigi, A., Penegini, M.: Shimura curves in the Prym locus. Commun. Contemp. Math. 21(2), 1850009 (2019) 
6. Eskin, A., McMullen, C.T., Mukamel, R.E., Wright, A.: Billiards, quadrilaterals, and moduli spaces. J. Am. Math. Soc. 33(4), 1039-1086 (2020)

7. Catanese, F.: Topological methods in moduli theory. Bull. Math. Sci. 5, 287-449 (2015)

8. Frapporti, D, Gleißner, C:: On threefolds isogenous to a product of curves. J. Algebra 465, 170-189 (2016)

9. Catanese, F., Lönne, M., Perroni, F. Kodaira fibrations and beyond: methods for moduli theory. Jpn. J. Math. 12(2), 91-174 (2017)

10. Lee, J.A., Lönne, M., Rollenske, S.: Double Kodaira fibrations with small signature. Int. J. Math. 31(7) (2020)

11. Li, B.: $G$-marked moduli spaces. Commun. Contemp. Math. 20(6), 1750061 (2018)

12. Catanese, F.: Genus stabilization for the components of moduli spaces of curves with symmetries. Algebr. Geom. 3(1), 23-49 (2016)

13. Kresch, A., Vistoli, A.: On coverings of Deligne-Mumford stacks and surjectivity of the Brauer map. Bull. Lond. Math. Soc. 36(2), 188-192 (2004)

14. Arbarello, E., Cornalba, M.: Teichmüller space via Kuranishi families. Ann. Sci. Norm. Super. Pisa Cl. Sci. 8(1), 89-116 (2009)

15. Arbarello, E., Cornalba, M., Griffiths, P.A.: Geometry of algebraic curves. Volume II, Grundlehren der Mathematischen Wissenschaften [Fundamental Principles of Mathematical Sciences], vol. 268. Springer, Heidelberg (2011)

16. Kerckhoff, S.P.: The Nielsen realization problem. Ann. Math. 117(2), 235-265 (1983)

17. Adem, A., Leida, J., Ruan, Y.: Orbifolds and stringy topology, Cambridge Tracts in Mathematics, vol. 171. Cambridge University Press, Cambridge (2007)

18. Harvey, W.J.: On branch loci in Teichmüller space. Trans. Am. Math. Soc. 153, 387-399 (1971)

19. Catanese, F.: Fibred surfaces, varieties isogenous to a product and related moduli spaces. Am. J. Math. 122, 1-44 (2000)

20. González Díez, G., Harvey, W.J.: Moduli of Riemann Surfaces with Symmetry, Discrete Groups and Geometry (Birmingham: London) Math. Soc. Lecture Note Ser., vol. 173, pp. 75-93. Cambridge University Press, Cambridge (1991) (1992)

21. Catanese, F., Lönne, M., Perroni, F. Homological stability for moduli spaces of curves with symmetries (2020) (work in progress)

22. Grauert, H., Remmert, R.: Komplexe Räume. Math. Ann. 136, 245-318 (1958)

23. Hartshorne, R.: Algebraic geometry. In: Graduate Texts in Math. no. 52, Springer, New York (1977)

24. Laumon, G., Moret-Bailly, L.: Champs algébriques, Ergebnisse der Mathematik und ihrer Grenzgebiete. 3. Folge. A Series of Modern Surveys in Mathematics [Results in Mathematics and Related Areas. 3rd Series. A Series of Modern Surveys in Mathematics], vol. 39. Springer, Berlin (2000)

Publisher's Note Springer Nature remains neutral with regard to jurisdictional claims in published maps and institutional affiliations. 